Imperial/TP/2006/DR/01

\title{
Popescu-Rohrlich Boxes in Quantum Measure Theory
}

\author{
Matthew Barnett, Fay Dowker and David Rideout \\ Blackett Laboratory, Imperial College \\ London, SWr 2AZ, U.K.
}

Dedicated to Rafael Sorkin on the occasion of his 60th birthday.

\begin{abstract}
Two results are proved at the quantal level in Sorkin's hierarchy of measure theories. One is a strengthening of an existing bound on the correlations in the EPR-Bohm setup under the assumption that the probabilities admit a strongly positive joint quantal measure. It is also proved that any set of no-signalling probabilities, for two distant experimenters with a choice of two alternative experiments each and two possible outcomes per experiment, admits a joint quantal measure, though one that is not necessarily strongly positive.
\end{abstract}




\section{Introduction}

An answer to the question "What is the essential nature of a quantum theory?" has been proposed by Rafael Sorkin. A quantum theory, according to Sorkin's scheme of generalised measure theories, is one in which pairs of alternative spacetime histories of a system can interfere but in which there is no interference among triples of histories that is not already accounted for by the pairwise interference [1. All quantum theories, by this criterion, are placed at the second level in a hierarchy of measure theories: a level $k$ theory allows interference among $k$-tuples of histories but no irreducible interference among $(k+1)$-tuples. Classical stochastic theories (and deterministic theories as special cases) are contained at level 1.

The surprising thing about this scheme is that the class of quantum theories includes all standard unitary quantum theories but is by no means exhausted by them. There are generalisations of the standard theory which yet remain at level 2. Even more surprisingly, one of these generalisations has been shown [2] to include the device that has come to be known as the "Popescu Rohrlich (PR) box" [3, 4].

Our purpose here is to investigate the structure of quantum measure theory including the way that PR boxes fit into it. We will concentrate on the situation we refer to as the $(2,2,2)$ case which is the standard EPR-Bohm experimental setup in which the ClauserHorne-Shimony-Holt-Bell (CHSHB) inequalities are derived: 2 distant experimenters Alya and Bai, each have a choice of 2 alternative settings for their experiment and each experiment yields one of 2 possible macroscopic outcomes.

In section 2 we review the basic aspects of Sorkin's hierarchy of measure theories. We focus on level 2, the quantal level, which is closely related to Hartle's generalised quantum mechanics [5, 6] without the requirement of decoherence or "consistency". We review the properties of a decoherence functional and define the concept of strong positivity for quantal measures/decoherence functionals. In section 3 we show that there is a set of inequalities on the correlations that is implied by the existence of a strongly positive joint decoherence functional or quantal measure on the (fictitious) joint sample space of all possible outcomes of both of Alya's and both of Bai's experiments. This tightens the bounds derived in reference 2].

In section 4 we prove our main result that any system of no-signalling experimental probabilities in our $(2,2,2)$ set-up admits a joint quantal measure. In section 5 we conclude with tentative conjectures on more complicated set-ups, the corresponding PR-type boxes and higher levels in the Sorkin hierarchy.

\section{Generalised measure theory}

We introduce the hierarchy of measure theories and refer to references [1, 7, 8] for details. In a generalized measure theory, there is a sample space $\Omega$ of possible histories for the system in question. Normally these are to be thought of as "fine grained histories", meaning as complete a description of physical reality as is conceivable in the theory, e.g. for $n$-particle mechanics a history would be a set of $n$ trajectories, and for a scalar field theory, a history would be a field configuration on spacetime. Predictions about the system - the dynamical 
content of the theory - are to be gleaned, in some way or another, from a (generalized) measure $\mu$, a non-negative real function on subsets of $\Omega$ (strictly, on some suitable class of "measurable" subsets of $\Omega$ ).

Given the measure, we can construct the following series of symmetric set functions:

$$
\begin{aligned}
I_{1}(X) \equiv & \mu(X) \\
I_{2}(X, Y) \equiv & \mu(X \sqcup Y)-\mu(X)-\mu(Y) \\
I_{3}(X, Y, Z) \equiv & \mu(X \sqcup Y \sqcup Z)-\mu(X \sqcup Y)-\mu(Y \sqcup Z)-\mu(Z \sqcup X) \\
& +\mu(X)+\mu(Y)+\mu(Z)
\end{aligned}
$$

and so on, where $X, Y, Z$, etc. are disjoint subsets of $\Omega$, as indicated by the symbol ' $\sqcup$ ' for disjoint union.

A measure theory of level $k$ is defined as one which satisfies the sum rule $I_{k+1}=0$. This condition implies that all higher sum rules are automatically satisfied, viz. $I_{k+n}=0$ for all $n \geq 1$. This means that each level of the hierarchy contains all the levels below it. A level 1 theory is one in which the measure satisfies the usual Kolmogorov sum rules of classical probability theory, classical Brownian motion being a good example. A level 2 theory is one in which the Kolmogorov sum rules may be violated but $I_{3}$ is nevertheless zero. Unitary quantum mechanics satisfies this condition and so we call level 2 generally the level of quantum measure theory.

The existence of a normalized quantal measure on $\Omega$ is equivalent [1] to the existence of a decoherence functional $D(X ; Y)$ of pairs of subsets of $\Omega$ satisfying [5, 6]:

(i) Hermiticity: $D(X ; Y)=D(Y ; X)^{*}, \forall X, Y$;

(ii) Additivity: $D(X \sqcup Y ; Z)=D(X ; Z)+D(Y ; Z), \forall X, Y, Z$ with $X$ and $Y$ disjoint;

(iii) Positivity: $D(X ; X) \geq 0, \forall X$;

(iv) Normalization: $D(\Omega ; \Omega)=1$.

The quantal measure is given by a decoherence functional via

$$
\mu(X)=D(X ; X)
$$

The quantity $D(X ; Y)$ is interpretable as the quantum interference between two sets of histories in the case when they are disjoint. Notice that $\mu$ determines only the real part of $D$. To see how the decoherence functional is defined for ordinary non-relativistic particle quantum mechanics see for example [6, 9].

A further condition on a decoherence functional is the condition of strong positivity, which states that for any finite collection of (not necessarily disjoint) subsets $X_{1}, X_{2}, \ldots X_{n}$ of $\Omega$, the $n \times n$ matrix $M_{i j} \equiv D\left(X_{i} ; X_{j}\right)$ is positive semidefinite. The decoherence functional of any ordinary unitary quantum mechanical theory is strongly positive. So is the decoherence functional for any open quantum system that is derived from a unitary model by summing out over some ignored variables. The only concrete dynamical decoherence functionals known to us that are not derivative of an underlying unitary model are those for the family of "quantum random walks" of Martin et al [10] and these are also strongly positive.

Strong positivity is a powerful requirement because it implies that there is a Hilbert space associated with the quantum measure, which turns out to be the standard Hilbert space in the case of unitary quantum mechanics [10, 11]. Strong positivity plays an important role in 
the present investigation as will be made clear. Indeed it will be shown that the question of why there are (apparently) no PR boxes in nature becomes, in the quantum measure theory framework, the question of why the decoherence functional is strongly positive.

We will not enter into the general question of how to interpret the quantal measure. Suffice it to mention that one set of ideas for doing so goes by the name of "consistent histories" or "decoherent histories" and attempts in effect to reduce the quantal measure to a classical one by the imposition of decoherence conditions [12, 13, 14, 6]. And a different attempt at an interpretation, in which the microscopic world is just as real as the macroscopic, may be found in [7. For our purposes in this paper, it will be enough to assume, where macroscopic measuring instruments are concerned, that distinct "pointer readings" do not interfere and that their quantal measures can be interpreted as probabilities in the sense of frequencies.

\section{The Tsirelson inequalities, I, II and III}

We follow 2] in setting up the following notation. Consider two distant experimenters, Alya and Bai, who each perform one of two possible experiments: Alya chooses setting $a$ or $a^{\prime}$ for her experiment, and Bai chooses setting $b$ or $b^{\prime}$ for his. Each then obtains one of two possible outcomes, +1 or -1 . This means, that, a priori, one has four entirely distinct probability distributions, $\mathbb{P}_{\alpha \beta}$ each defined on its own four-element sample space $\Omega_{\alpha \beta}=\Omega_{\alpha} \times \Omega_{\beta}$, where $\alpha$ ranges over the settings $a$ or $a^{\prime}, \beta$ ranges over the settings $b$ or $b^{\prime}$, and each space $\Omega_{\alpha}$, $\Omega_{\beta}$ is a binary sample space, corresponding to the two possible experimental outcomes, \pm 1 . We refer to the four probability distributions, $\mathbb{P}_{a b}, \mathbb{P}_{a b^{\prime}}, \mathbb{P}_{a^{\prime} b}$ and $\mathbb{P}_{a^{\prime} b^{\prime}}$, together as a system of experimental probabilities. We will make the assumption throughout that every system of experimental probabilities satisfies the "no-signalling" conditions, namely, the marginal probabilities for the outcomes of Alya's a experiment are the same whether calculated from $\mathbb{P}_{a b}$ or $\mathbb{P}_{a b^{\prime}}$ and similarly for her $a^{\prime}$ experiment and similarly for Bai's experiments.

Now we merge the $\Omega_{\alpha \beta}$ into a single sample space

$$
\Omega \equiv \Omega_{a} \times \Omega_{a^{\prime}} \times \Omega_{b} \times \Omega_{b^{\prime}}
$$

of $2^{4}=16$ elements. Let us label the elements of $\Omega$ by the sixteen 4-element bit strings $\left\{\left(i i^{\prime} j j^{\prime}\right): i, i^{\prime}, j, j^{\prime}= \pm 1\right\}$ where $i$ corresponds to the $a$ outcome, $i^{\prime}$ to $a^{\prime}, j$ to $b$ and $j^{\prime}$ to $b^{\prime}$.

We say that the experimental probabilities admit a joint quantal measure iff there exists a decoherence functional $D$ on $\Omega$ such that its marginals agree with the experimental probabilities in the following way

$$
\begin{aligned}
\sum_{i^{\prime} j^{\prime} k^{\prime} l^{\prime}} D\left(i i^{\prime} j j^{\prime} ; k k^{\prime} l l^{\prime}\right) & =\mathbb{P}_{a b}(i j) \delta_{i k} \delta_{j l}, \\
\sum_{i^{\prime} j k^{\prime} l} D\left(i i^{\prime} j j^{\prime} ; k k^{\prime} l l^{\prime}\right) & =\mathbb{P}_{a b^{\prime}}\left(i j^{\prime}\right) \delta_{i k} \delta_{j^{\prime} l^{\prime}} \\
\sum_{i j^{\prime} k l^{\prime}} D\left(i i^{\prime} j j^{\prime} ; k k^{\prime} l l^{\prime}\right) & =\mathbb{P}_{a^{\prime} b}\left(i^{\prime} j\right) \delta_{i^{\prime} k^{\prime}} \delta_{j l}, \\
\sum_{i j k l} D\left(i i^{\prime} j j^{\prime} ; k k^{\prime} l l^{\prime}\right) & =\mathbb{P}_{a^{\prime} b^{\prime}}\left(i^{\prime} j^{\prime}\right) \delta_{i^{\prime} k^{\prime}} \delta_{j^{\prime} l^{\prime}} .
\end{aligned}
$$


If there exists such a joint decoherence functional that is moreover strongly positive we say that the experimental probabilities admit a strongly positive joint quantal measure (SPJQM).

Note that the marginal decoherence functionals, one for each realizable pair of experiments, are required to decohere on the outcomes.

It was shown in 2] that certain inequalities on the correlations, known as the Tsirelson inequalities [15, follow from the existence of a SPJQM. If we define $X_{\alpha \beta}$ to be the "correlator" for the $(\alpha, \beta)$ experiment via:

$$
\begin{aligned}
X_{a b} & =\sum_{i j} i j \mathbb{P}_{a b}(i j) \\
X_{a b^{\prime}} & =\sum_{i j^{\prime}} i j^{\prime} \mathbb{P}_{a b^{\prime}}\left(i j^{\prime}\right) \\
X_{a^{\prime} b} & =\sum_{i^{\prime} j} i^{\prime} j \mathbb{P}_{a^{\prime} b}\left(i^{\prime} j\right) \\
X_{a^{\prime} b^{\prime}} & =\sum_{i^{\prime} j^{\prime}} i^{\prime} j^{\prime} \mathbb{P}_{a^{\prime} b^{\prime}}\left(i^{\prime} j^{\prime}\right)
\end{aligned}
$$

then the inequalities are

$$
\left|X_{a b}+X_{a^{\prime} b}+X_{a b^{\prime}}-X_{a^{\prime} b^{\prime}}\right| \leq 2 \sqrt{2}
$$

plus the three other inequalities where the minus sign is given to each of the other $X$ 's in turn. We will refer to these inequalities as TsirelsonI. The CHSHB inequalities have the same form except the $2 \sqrt{2}$ is replaced by 2 .

We now improve these bounds to stronger inequalities. First, as described in [2], we apply to $D$ a certain basic construction via which any strongly positive decoherence functional gives rise to a Hilbert space [10, 11. Indeed, if the experimental probabilities admit a SPJQM, via a decoherence functional $D$ as above, then there is a Hilbert space $\mathcal{H}$ spanned by vectors $\left\{\left|i i^{\prime} j j^{\prime}\right\rangle\right\}$ labelled by the elements of $\Omega$ and on which the inner product is given by

$$
\left\langle i i^{\prime} j j^{\prime} \mid k k^{\prime} l l^{\prime}\right\rangle=D\left(i i^{\prime} j j^{\prime} ; k k^{\prime} l l^{\prime}\right) \text {. }
$$

We will require the following Lemma, the proof of which is given in [2].

Lemma 1. Let the experimental probabilities admit a SPJQM given by the decoherence functional $D$ on $\Omega$. Let $|a\rangle,\left|a^{\prime}\right\rangle$, $|b\rangle$, and $\left|b^{\prime}\right\rangle \in \mathcal{H}$ be defined by

$$
\begin{aligned}
|a\rangle & =\sum_{i i^{\prime} j j^{\prime}} i\left|i i^{\prime} j j^{\prime}\right\rangle, \\
\left|a^{\prime}\right\rangle & =\sum_{i i^{\prime} j j^{\prime}} i^{\prime}\left|i i^{\prime} j j^{\prime}\right\rangle, \\
|b\rangle & =\sum_{i i^{\prime} j j^{\prime}} j\left|i i^{\prime} j j^{\prime}\right\rangle, \\
\left|b^{\prime}\right\rangle & =\sum_{i i^{\prime} j j^{\prime}} j^{\prime}\left|i i^{\prime} j j^{\prime}\right\rangle .
\end{aligned}
$$


Then

$$
\langle a \mid a\rangle=\langle b \mid b\rangle=\left\langle a^{\prime} \mid a^{\prime}\right\rangle=\left\langle b^{\prime} \mid b^{\prime}\right\rangle=1
$$

and

$$
\langle a \mid b\rangle=X_{a b},\left\langle a \mid b^{\prime}\right\rangle=X_{a b^{\prime}},\left\langle a^{\prime} \mid b\right\rangle=X_{a^{\prime} b},\left\langle a^{\prime} \mid b^{\prime}\right\rangle=X_{a^{\prime} b^{\prime}} .
$$

We are now ready to prove our first result.

Theorem 1. If a system of experimental probabilities for the (2,2,2) system admits a SPJQM then

$$
\left|\arcsin X_{a b}+\arcsin X_{a^{\prime} b}+\arcsin X_{a b^{\prime}}-\arcsin X_{a^{\prime} b^{\prime}}\right| \leq \pi
$$

where each angle, $\arcsin X_{\alpha \beta}$, lies between $-\pi / 2$ and $\pi / 2$.

Proof. It suffices to prove (3.5) without the absolute value signs as one sees by reversing the signs of Bai's outcomes.

Defining the angles

$$
\begin{aligned}
\theta_{1} & =\frac{\pi}{2}-\arcsin X_{a b} \\
\theta_{2} & =\frac{\pi}{2}-\arcsin X_{a b^{\prime}} \\
\theta_{3} & =\frac{\pi}{2}-\arcsin X_{a^{\prime} b} \\
\theta_{4} & =\frac{\pi}{2}-\arcsin X_{a^{\prime} b^{\prime}}
\end{aligned}
$$

one sees that by Lemma 1

$$
\cos \theta_{1}=<a\left|b>, \cos \theta_{2}=<a\right| b^{\prime}>, \cos \theta_{3}=<a^{\prime}\left|b>, \cos \theta_{4}=<a^{\prime}\right| b^{\prime}>
$$

and the inequality to be proved boils down to an inequality on angles between 4 unit vectors in a real vector space.

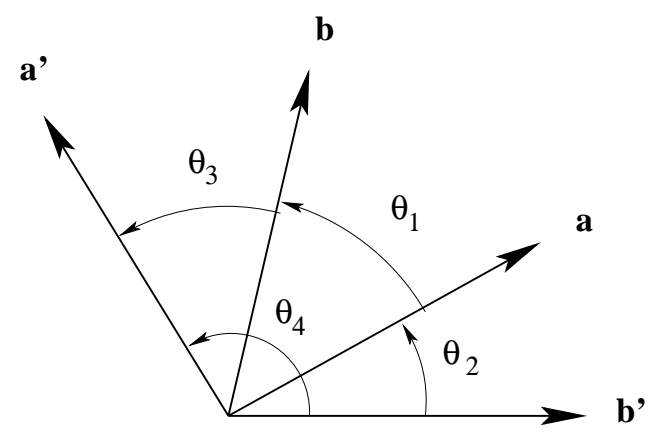

Figure 1: Four coplanar vectors, $\mathbf{a}, \mathbf{a}^{\prime}, \mathbf{b}$ and $\mathbf{b}^{\prime}$.

Suppose $\theta_{1}+\theta_{2}+\theta_{3} \leq \pi$. Then if all four vectors are coplanar, $\theta_{4}=\theta_{1}+\theta_{2}+\theta_{3}$ (see figure 1 ) and if they are not coplanar, $\theta_{4} \leq \theta_{1}+\theta_{2}+\theta_{3}$. If $\theta_{1}+\theta_{2}+\theta_{3}>\pi$ then $\theta_{1}+\theta_{2}+\theta_{3}-\theta_{4} \geq 0$, since $\theta_{4} \leq \pi$. In either case we have

$$
-\theta_{1}-\theta_{2}-\theta_{3}+\theta_{4} \leq 0
$$

and hence the result. 
The other three inequalities gotten from (3.5) by moving the minus sign in front of the other correlators in turn are proved similarly and we refer to them all collectively as TsirelsonII.

\subsection{Relationship to Ordinary Quantum Mechanics}

Tsirelson proved that TsirelsonII are necessary and sufficient conditions for the existence of what we will call an "ordinary quantum model for the correlators" (OQMC) 16, 17. An OQMC consists of a vector $|\psi\rangle$ in a Hilbert space, and two pairs of self-adjoint operators $\left\{S^{a}, S^{a^{\prime}}\right\}$ and $\left\{S^{b}, S^{b^{\prime}}\right\}$ such that $S^{\alpha}$ commutes with $S^{\beta}$, for all $\alpha$ and $\beta$, and such that

$$
X_{\alpha \beta}=<\psi\left|S^{\alpha} S^{\beta}\right| \psi>\text {, }
$$

for all $\alpha$ and $\beta$.

TsirelsonII are necessary but not sufficient for the existence of an "ordinary quantum model for the probabilities" (OQMP) however. Such an OQMP would consist of a vector $\mid \psi>$ and two pairs of projective decompositions of unity, $\left\{P_{a}^{+}, P_{a}^{-}\right\},\left\{P_{a^{\prime}}^{+}, P_{a^{\prime}}^{-}\right\},\left\{P_{b}^{+}, P_{b}^{-}\right\}$ and $\left\{P_{b^{\prime}}^{+}, P_{b^{\prime}}^{-}\right\}$, such that $\left[P_{\alpha}^{i}, P_{\beta}^{j}\right]=0$ and

$$
\mathbb{P}_{\alpha \beta}(i j)=<\psi\left|P_{\alpha}^{i} P_{\beta}^{j}\right| \psi>
$$

for all $\alpha$ and $\beta$, and all $i$ and $j$. The necessary and sufficient conditions for an OQMP to exist are given by Tsirelson in reference [15] and we refer to them as TsirelsonIII.

We know that if an OQMP exists then there exists a SPJDF [2]. It is not known whether the converse is true.

\section{PR Boxes}

The device that has come to be known as a PR box is a box with two knobs - "A" and "B" each with two settings $-a$ or $a^{\prime}$ for $A$ and $b$ or $b^{\prime}$ for $B$ - which may be thought of as inputs. For each setting $(\alpha, \beta)$ for the box, it produces two outputs, \pm 1 for $A$ and \pm 1 for $B$. So the setup is simply that of the EPR-Bohm experiment and we can employ our notation from above. The interesting features of the PR box are that the probability distributions on the outcomes given the settings (i.e. the experimental probabilities in our previous language) maximally violate one of the CHSHB inequalities and moreover violate TsirelsonII and so do not admit an OQMC/OQMP. Noting that each correlator, $X_{\alpha \beta}$ lies between -1 and 1 , the "CHSH" function $Q \equiv X_{a b}+X_{a b^{\prime}}+X_{a^{\prime} b^{\prime}}-X_{a^{\prime} b^{\prime}}$ has a "logical" upper bound of 4 .

There is a unique set of experimental probabilities which gives rise to correlators which saturate this logical bound: $X_{a b}=X_{a b^{\prime}}=X_{a^{\prime} b^{\prime}}=1$ and $X_{a^{\prime} b^{\prime}}=-1$. These are

$$
\begin{gathered}
\mathbb{P}_{a b}(+1,+1)=\mathbb{P}_{a b}(-1,-1)=\frac{1}{2} \\
\mathbb{P}_{a b^{\prime}}(+1,+1)=\mathbb{P}_{a b^{\prime}}(-1,-1)=\frac{1}{2} \\
\mathbb{P}_{a^{\prime} b}(+1,+1)=\mathbb{P}_{a^{\prime} b}(-1,-1)=\frac{1}{2} \\
\mathbb{P}_{a^{\prime} b^{\prime}}(+1,-1)=\mathbb{P}_{a^{\prime} b^{\prime}}(-1,+1)=\frac{1}{2}
\end{gathered}
$$


There are 7 other PR boxes, obtainable from this one by local operations: Alya can flip each of her inputs or outputs and so can Bai [18]. Each PR box maximally violates one of the 8 CHSH inequalities.

In [2] it was shown that the PR box admits a joint quantal measure, indeed a decoherence functional that does the job for one of the PR boxes is explicitly given in that paper. By doing the appropriate bit-flip on that decoherence functional (either flip the outcome of the $a^{\prime}$ measurement or swap $b$ and $b^{\prime}$ : both produce the same result), we obtain the decoherence functional for the PR box (4.1):

$$
\begin{aligned}
D_{P R}(-+--;-+--) & =D_{P R}(+++-;+++-)=D_{P R}(++-+;++-+) \\
& =D_{P R}(---+;---+)=\frac{1}{2} \\
-D_{P R}(-+-+;-+--) & =-D_{P R}(+++-;++--)=D_{P R}(---+;----) \\
& =D_{P R}(-+-+;+---)=-D_{P R}(++-+;+---) \\
& =-D_{P R}(---+;+---)=D_{P R}(++-+;+++-) \\
& =-D_{P R}(++-+;-+-+)=-D_{P R}(---+;-+-+) \\
& =D_{P R}(---+;++-+)=\frac{1}{4}
\end{aligned}
$$

The remaining elements which are not equal to one of the above by Hermiticity are zero.

Since the correlators violate TsirelsonII the result of [2] shows that the measure is not strongly positive but it is nevertheless quantal, according to the Sorkin classification. The joint quantal measures for the other PR boxes are obtained similarly by performing appropriate bit-flips.

Theorem 2. Any system of (no-signalling) experimental probabilities for the (2,2,2) setup admits a joint quantal measure.

Proof. A system of experimental probabilities for the $(2,2,2)$ setup is a vector $\mathbf{y}$ in an eight dimensional real vector space because there are only eight independent probabilities, the rest being fixed by the no-signalling conditions and normalisation. $\mathbf{y}$ is an element of a polytope whose 24 vertices, $\mathbf{v}_{i}, i=1, \ldots 24$, consist of the 8 PR boxes and $2^{4}=16$ so-called "local" vertices which are the deterministic boxes in which each of Alya's and Bai's inputs has a definite output 18. In other words, $\mathbf{y}$ is a convex combination of these vertices, $\mathbf{y}=\sum_{i=1}^{24} p_{i} \mathbf{v}_{i}$ with $\sum_{i=1}^{24} p_{i}=1$ and all $p_{i} \geq 0$.

Each of the 8 PR boxes admits a joint quantal measure.

Each local vertex admits a joint probability (level 1) measure: the probability of exactly one of the histories in $\Omega$ - the one that corresponds to the deterministic box - is 1 and the other histories have zero probability. A probability measure is a special case of a joint quantal measure: each level of the hierarchy includes the lower levels.

So each vertex of the polytope admits a joint quantal measure $D_{i}(\cdot ; \cdot)$ and therefore the measure $D(\cdot ; \cdot)=\sum_{i=1}^{24} p_{i} D_{i}(\cdot ; \cdot)$ is a joint quantal measure for $\mathbf{y}$. The only condition that might be violated is positivity but this follows from the convexity: a convex combination of non-negative numbers is non-negative. 


\section{Discussion}

The question of whether the existence of a SPJQM implies the existence of a OQMP remains open. One could try to find a counterexample by finding a set of probabilities on the boundary of the region in the polytope of probabilities which allows a OQMP. That point will admit a SPJQM. One could try to find perturbations that take it outside the OQMP region whilst preserving the strong positivity of the decoherence functional. The difficulty of this strategy lies in the sheer complexity of Tsirelson's conditions for the existence of an OQMP [15]. A counterexample - a set of experimental probabilities that does not admit an OQMP but which does admit a SPJDF - would be extremely interesting because it would mean that experiment could in principle distinguish between "ordinary quantum theory" and "quantum measure theory plus strong positivity".

The question posed by Popescu and Rohrlich and others, "Why does nature [apparently] not make use of the full power of possible non-local non-signalling correlations by realising a physical PR box?" ${ }^{1}$ is transformed, in the framework of generalised measure theory, into two questions. Why does nature (apparently) not make use of level 3 and higher of the hierarchy of measure theories and why, if it does indeed restrict itself to the quantal level, is strong positivity (apparently) realised.

One answer to the former question could be provided if it were the case that any set of no-signalling probabilities for any number of experimenters, settings (depending on experimenter) and outcomes (depending on experimenter and setting) admitted a joint quantal measure. Then one could argue that nature doesn't use the level 3 measures because there is no need: one could always concoct a level 2 measure to simulate any finite set of experimental data. We are sceptical about this possibility but it needs to be investigated. This can be done by studying correlated probability distributions that have the potential of involving irreducible three-history interference. Such three-history interference would show itself in situations involving either three settings for at least one experimenter, or three outcomes for at least one experimenter-and-setting. We suspect that outcomes in such situations can be correlated in ways that cannot be accounted for at level 2 , but can be accommodated at level 3. For example, we conjecture that the $P R_{n}$ box introduced in [20] does not admit a joint level $n-1$ measure but does admit a joint level $n$ measure, $n \geq 2$.

Even within the context of level 2 measures, there remains the question of strong positivity which is discussed in some detail in reference [10]. The main reason that the condition is adopted in that paper is calculational feasibility. Positivity of the decoherence functional for the quantal random walk is guaranteed by strong positivity which in turn is guaranteed by positivity of the "transfer matrix" which propagates the decoherence functional from one time step to the next. This is relatively easy to satisfy. The condition of positivity on the other hand is extremely difficult to implement without imposing strong positivity. At each time step there are $2^{N}-1$ positivity conditions (one for each non-empty subset of the sample space) to satisfy where $N$ is the number of distinct histories to that time, and these conditions do not seem to translate into any simple condition on the transfer matrix. Given that the cardinality of the sample space of possible paths grows exponentially with discrete

\footnotetext{
${ }^{1}$ Ref. [19] claims to realize a PR box within ordinary quantum theory by restricting to a final state. However, the measurement outcomes no longer decohere in this case, and thus the physical interpretation of the setup is obscured.
} 
time, the number of positivity conditions grows as an exponential of an exponential in time.

Strong positivity allows the construction of a Hilbert space from the decoherence functional. Moreover it guarantees that formally combining two non-interacting subsystems into a single system does not spoil positivity [10]: if the individual subsystems were only positive and not strongly positive, the combined system need not be positive. Are these strong enough physical arguments for strong positivity?

\section{Acknowledgements}

We thank Jonathan Halliwell, Joe Henson, Rafael Sorkin, Jamie Vicary, Hans Westman and the members of the Imperial College Relativity lunch for useful discussions. This work was supported in part by the Marie Curie Research and Training Network ENRAGE (MRTNCT-2004-005616).

\section{References}

[1] R. D. Sorkin, Quantum mechanics as quantum measure theory, Mod. Phys. Lett. A9 (1994) 3119-3128, gr-qc/9401003.

[2] D. Craig, F. Dowker, J. Henson, S. Major, D. Rideout, and R. Sorkin, A Bell inequality analog in quantum measure theory, 2006.

[3] L. A. Khalfin and B. S. Tsirelson in Symposium on the Foundations of Modern Physics (P. Lahti and P. Mittelstædt, eds.), pp. 441-460, World Scientific, Singapore, 1985.

[4] S. Popescu and D. Rohrlich, Nonlocality as an axiom, Found. Phys. 24 (1994) 379.

[5] J. B. Hartle, The quantum mechanics of cosmology, in Quantum Cosmology and Baby Universes: Proceedings of the 1989 Jerusalem Winter School for Theoretical Physics (S. Coleman, J. B. Hartle, T. Piran, and S. Weinberg, eds.), World Scientific, Singapore, 1991.

[6] J. B. Hartle, Space-time quantum mechanics and the quantum mechanics of space-time, in Proceedings of the Les Houches Summer School on Gravitation and Quantizations, Les Houches, France, 6 Jul - 1 Aug 1992 (J. Zinn-Justin and B. Julia, eds.), North-Holland, 1995. gr-qc/9304006.

[7] R. D. Sorkin, Quantum measure theory and its interpretation, in Quantum Classical Correspondence: Proceedings of 4th Drexel Symposium on Quantum Nonintegrability, September 8-11 1994, Philadelphia, PA (D. Feng and B.-L. Hu, eds.), pp. 229-251, International Press, Cambridge, Mass., 1997. gr-qc/9507057.

[8] R. B. Salgado, Some identities for the quantum measure and its generalizations, Mod. Phys. Lett. A17 (2002) 711-728, gr-qc/9903015. 
[9] J. B. Hartle, Generalizing quantum mechanics for quantum spacetime, gr-qc/0602013. Contribution to the 23rd Solvay Conference, The Quantum Structure of Space and Time.

[10] X. Martin, D. O'Connor, and R. D. Sorkin, The random walk in generalized quantum theory, Phys. Rev. D71 (2005) 024029, gr-qc/0403085.

[11] F. Dowker, R. Garcia, and R. D. Sorkin, Hilbert space from quantum measure theory, 2005. in preparation.

[12] R. B. Griffiths, Consistent histories and the interpretation of quantum mechanics, J. Statist. Phys. 36 (1984) 219-272.

[13] R. Omnès, Logical reformulation of quantum mechanics. 1. Foundations, J. Stat. Phys. 53 (1988) 893-932.

[14] M. Gell-Mann and J. B. Hartle, Quantum mechanics in the light of quantum cosmology, in Complexity, Entropy and the Physics of Information, SFI Studies in the Sciences of Complexity, Vol VIII (W. Zurek, ed.), pp. 150-173, Addison Wesley, Reading, 1990.

[15] B. Cirel'son, Quantum generalisations of Bell's inequality, Lett. Math. Phys. 4 (1980) 93-100.

[16] B. Tsirel'son, Quantum analogues of the bell inequalities. the case of two spatially separated domains, J. Soviet Math. 36 (1987) 557-570. Translated from a source in Russian of 1985.

[17] B. Tsirelson, Some results and problems on quantum bell-type inequalities, Hadronic Journal Supplement 8 (1993) 329-345.

[18] J. Barrett, N. Linden, S. Massar, S. Pironio, S. Popescu, and D. Roberts, Non-local correlations as an information theoretic resource, Physical Review A 71 (2005) 022101.

[19] S. Marcovitch, B. Reznik, and L. Vaidman, Quantum mechanical realization of a "PR-box", quant-ph/0601122.

[20] N. Brunner, V. Scarani, and N. Gisin, Bell-type inequalities for non-local resources, quant-ph/0603094. 